\title{
Microbe-ID: An open source toolbox for microbial genotyping and species identification
}

\author{
Javier F Tabima ${ }^{1}$, Sydney E Everhart ${ }^{1,2}{ }^{\text {， Meredith M Larsen }}{ }^{3}$, Alexandra J Weisberg ${ }^{1}$, Zhian N Kamvar $^{1}$,

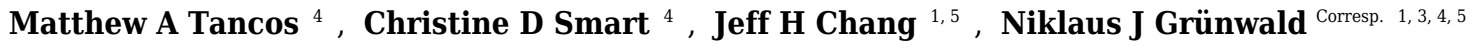 \\ 1 Department of Botany and Plant Pathology, Oregon State University, Corvallis, OR, United States \\ 2 Department of Plant Pathology, University of Nebraska, Lincoln, NE, United States \\ 3 Horticultural Crops Research Laboratory, USDA Agricultural Research Service, Corvallis, OR, United States \\ 4 Plant Pathology and Plant-Microbe Biology Section, School of Integrative Plant Science, Cornell University, Geneva, NY, United States \\ 5 Molecular and Cellular Biology Graduate Program and Center for Genome Biology and Biocomputing, Oregon State University, Corvallis, OR, United \\ States
}

Corresponding Author: Niklaus J Grünwald

Email address: nik.grunwald@ars.usda.gov

Development of tools to identify species, genotypes, or novel strains of invasive organisms is critical for monitoring emergence and implementing rapid response measures. Molecular markers, although critical to identifying species or genotypes, require bioinformatic tools for analysis. However, user-friendly analytical tools for fast identification are not readily available. To address this need, we created a web-based set of applications called Microbe-ID that allow for customizing a toolbox for rapid species identification and strain genotyping using any genetic markers of choice. Two components of Microbe-ID, named Sequence-ID and Genotype-ID, implement species and genotype identification, respectively. Sequence-ID allows identification of species by using BLAST to query sequences for any locus of interest against a custom reference sequence database. Genotype-ID allows placement of an unknown multilocus marker in either a minimum spanning network or dendrogram with bootstrap support from a user-created reference database. Microbe-ID can be used for identification of any organism based on nucleotide sequences or any molecular marker type and several examples are provided. We created a public website for demonstration purposes called Microbe-ID ( www.microbe-id.org) and provided a working implementation for the genus Phytophthora ( www.phytophthora-id.org ). In Phytophthora-ID, the Sequence-ID application allows identification based on ITS or cox spacer sequences. Genotype-ID groups individuals into clonal lineages based on simple sequence repeat (SSR) markers for the two invasive plant pathogen species $P$. infestans and $P$. ramorum. All code is open source and available on github and CRAN. Instructions for installation and use are provided at https://github.com/grunwaldlab/Microbe-ID. 
1 Microbe-ID: An open source toolbox for microbial genotyping and species identification 2

3 Javier F. Tabima ${ }^{1}$, Sydney E. Everhart ${ }^{1, \#}$, Meredith M. Larsen², Alexandra J. Weisberg ${ }^{1}$, Zhian

4 N. Kamvar ${ }^{1}$, Mathew A. Tancos ${ }^{3}$, Christine D. Smart ${ }^{3}$, Jeff H. Chang ${ }^{1,4}$, Niklaus J.

5 Grünwald $1,2,3,4$

6

$7 \quad{ }^{1}$ Department of Botany and Plant Pathology, Oregon State University, Corvallis, OR, USA

$8{ }^{2}$ Horticultural Crops Research Laboratory, USDA-ARS, Corvallis, OR, USA

$9{ }^{3}$ Plant Pathology and Plant-Microbe Biology Section, School of Integrative Plant Science,

10 Cornell University, Geneva, NY, USA

$11{ }^{4}$ Molecular and Cellular Biology Graduate Program and Center for Genome Biology and

12 Biocomputing, Oregon State University, Corvallis, OR, USA

14 \# Current address: Department of Plant Pathology, University of Nebraska, Lincoln, NE, USA

16 Corresponding Author:

17 Niklaus J. Grünwald ${ }^{1,2,3,4}$

183420 NW Orchard Ave., Corvallis, OR 97330, United States

19 E-mail address: nik.grunwald@ars.usda.gov 
20 Abstract

21

22 Development of tools to identify species, genotypes, or novel strains of invasive organisms is

23 critical for monitoring emergence and implementing rapid response measures. Molecular

24 markers, although critical to identifying species or genotypes, require bioinformatic tools for

25 analysis. However, user-friendly analytical tools for fast identification are not readily available.

26 To address this need, we created a web-based set of applications called Microbe-ID that allow

27 for customizing a toolbox for rapid species identification and strain genotyping using any genetic

28 markers of choice. Two components of Microbe-ID, named Sequence-ID and Genotype-ID,

29 implement species and genotype identification, respectively. Sequence-ID allows identification

30 of species by using BLAST to query sequences for any locus of interest against a custom

31 reference sequence database. Genotype-ID allows placement of an unknown multilocus marker

32 in either a minimum spanning network or dendrogram with bootstrap support from a user-created

33 reference database. Microbe-ID can be used for identification of any organism based on

34 nucleotide sequences or any molecular marker type and several examples are provided. We

35 created a public website for demonstration purposes called Microbe-ID (www.microbe-id.org)

36 and provided a working implementation for the genus Phytophthora (www.phytophthora-id.org).

37 In Phytophthora-ID, the Sequence-ID application allows identification based on ITS or cox

38 spacer sequences. Genotype-ID groups individuals into clonal lineages based on simple sequence

39 repeat (SSR) markers for the two invasive plant pathogen species P. infestans and P. ramorum.

40 All code is open source and available on github and CRAN. Instructions for installation and use

41 are provided at https://github.com/grunwaldlab/Microbe-ID. 


\section{Background}

Development of tools for identification of species, genotypes or strains is critical for monitoring emergence of invasive organisms such as Phytophthora ramorum causing sudden oak death (Grünwald et al. 2008), Hymenoscyphus fraxineus causing ash dieback (Gross et al. 2014), Aphanomyces astaci causing crayfish plague (Holdich et al. 2009), Cryptococcus gattii causing cryptococcosis and meningitis (Byrnes et al. 2010), or Methicillinresistant Staphylococcus aureus causing invasive MRSA disease (Klevens et al. 2007).

Molecular markers provide a rapid means for identification, but require various bioinformatics tools for identification of species and/or novel genotypes. In eukaryotes, sequences from the rRNA internal transcribed spacer (ITS) region and various mitochondrial DNA regions are used to separate discrete species (Coleman, 2003, 2007). ITS and mtDNA markers are now the most widely used markers in plants (Coleman, 2007, 2009), fungi (James et al., 2006), corals (Grajales et al., 2007), and oomycetes (Cooke et al., 2012; Robideau et al., 2011) and have been coined "DNA barcodes" because of their broad ability to distinguish species (Schoch et al., 2012). Classification of individuals using various molecular markers has recently increased. Multi-locus sequence types (MLST) are being widely used by researchers working with bacterial taxa to reveal the identity of samples by classification relative to known reference strains (Maiden et al., 2013). Other molecular markers or methods used to distinguish genotypes might include microsatellites (or simple sequence repeats) to identify strains and clonal lineages (Cooke et al., 2012; Ivors et al., 2006), DNA sequences for specific genic regions (Maiden et al., 2013), single nucleotide polymorphism (SNP) genotyping using reduced representation approaches (Grünwald 
65 et al. 2016) such as RAD-seq (Etter et al., 2010) or genotyping by sequencing (GBS, Elshire et

66 al., 2011), or genome wide SNP genotyping (Huang et al., 2009).

In addition to the molecular methods developed, different types of online databases have

been implemented to identify species using these molecular methods within groups of organisms. Examples of these databases are FungiDB for fungi and fungal-like organisms (Stajich et al., 2011), EuPathDB for eukaryotic organisms (Aurrecoechea et al., 2013), and the Phytophthora database which allows entries by experts in the Phytophthora community from different labs or countries for different species of the genus (Park et al., 2008). We previously reported on our development of a database for Phytophthora species and genotype identification using web tools to identify species using common barcodes, enabling the conjunction of modern laboratory techniques with highly curated databases for species identification (Grünwald et al. 2011). Our objective here was to report the development of a toolbox for microbe identification (Microbe-ID) that can readily be customized for sequence based species identification (Sequence-ID) or molecular marker-based identification of genotypes (Genotype-ID) for any group of organisms. Our objectives were two-fold: 1) to implement Microbe-ID as a demonstration site that is customizable for any group of organisms and 2) to demonstrate a working implementation at Phytophthora-ID.org version 2.0 with significant updates from version 1.0 (Grünwald et al. 2011). Microbe-ID includes two modules, Sequence-ID and Microbe-ID. Sequence analysis is implemented based on use of a well characterized barcode region for the genus Phytophthora, but can be implemented to use any barcode sequence of interest. Genotype analysis can be implemented to use a variety of marker data types. To demonstrate the breadth of the developed tools and applicability to the diversity of microorganisms, the following three examples were included: codominant microsatellite data 
88 (SSR/Microsatellite) for the oomycete P. ramorum (Grünwald et al. 2009), concatenated Multi

89 Locus Sequence Type (MLST) or individual locus sequences for the bacterium Clavibacter

90 michiganensis subsp. michiganensis (Tancos et al. 2015, Supplementary Figure 3), and dominant

91 Amplified Fragment Length Polymorphism (Binary (AFLP) data, Supplementary Figure 4) for

92 the oomycete Aphanomyces euteiches (Grünwald and Hoheisel, 2006). Moreover, Genotype-ID

93 can be expanded to include other marker systems including gene sequences for resistance to

94 antibiotics or fungicides as well as presence/absence polymorphisms for effector genes or other

95 adaptive loci. Two sequence databases were developed that help us demonstrate the utility of

96 Microbe-ID. These databases are for the genus Phytophthora (Phytophthora-ID) with two

97 sequence databases containing over 110 species that are mostly plant pathogens (Kroon et al.,

98 2012), and two genotyping databases for populations of the potato late blight pathogen, $P$.

99 infestans, and the sudden oak death pathogen, P. ramorum (Genotype-ID) (Grünwald et al. 2008;

100 Kamoun et al. 2015). All of these tools are readily customizable and open source

101 (http://www.github.com/grunwaldlab/microbe-ID), provided as a demonstration site

102 (http://microbe-id.org/), and a working implementation of Sequence-ID and Genotype-ID that we

103 use in our own work for the genus Phytophthora (www.phytophthora-id.org). Finally, a

104 companion paper describes application of Microbe-ID for the implementation of a new website,

105 Gall-ID, with novel tools for identification of gall-forming bacteria (Davis et al. 2016).

106

107 The Microbe-ID toolbox. We developed a template website named Microbe-ID

108 (http://microbe-id.org/) with two separate modules, Sequence-ID and Genotype-ID, for

109 sequence-based species identification and genotyping, respectively. The website is written using

110 bootstrap (http://getbootstrap.com/), a HTML, CSS, and JS framework for developing 
111 responsive, mobile first projects on the web. Specific instructions, code, and resources necessary

112 for implementation of Microbe-ID are provided on the github repository

113 (https://github.com/grunwaldlab/Microbe-ID). The server currently hosting Microbe-ID is

114 running Centos Linux release 6.6, NCBI BLAST 2.2.28+ (Altschul et al., 1990), MAFFT version

1157.221 (Katoh et al. 2002), and R version 3.1.2. Below we describe specific tools required for

116 customization of each module.

117 Navigating and using Microbe-ID. Navigating the demonstration site of Microbe-ID

118 (http://Microbe-ID.org) the user will encounter a menu bar with links to Sequence-ID, Genotype-

119 ID, and an about page. The home page has a general description of the functionality and

120 components implemented in Microbe-ID, as well as links to the github site. Since input from the

121 user is entered into forms as a query, each form implemented in Microbe-ID is encoded to check

122 that the format of the data supplied by the user is supported. If the format is incorrect, the page

123 will prompt the user with an error message, making the use of Microbe-ID more user-friendly.

124 Sequence-ID. We created a module called Sequence-ID that uses BLAST analysis of

125 common sequence loci for species identification (Supplementary Figure 1). Sequence-ID

126 includes a PERL_CGI script that permits the communication between a user interface form

127 (implemented in HTML5) and a BLAST database for the desired sequence data. The form

128 recognizes the input in FASTA format and uses BLASTN to search for the most similar DNA

129 sequences in the marker database. The PERL-CGI script receives the information of the HTML

130 tabulated output from BLAST and displays a table of hits to the end user. Sequence-ID is

131 customizable as the FASTA data file can readily be updated using the makeblastdb program of

132 the BLAST suite. It can also similarly be implemented for BLASTP analysis of amino acid

133 sequences. 

web-app is contained, and a "Help" tab which contains a link to a "Site Help" prompt. This "Site

136 Help" prompt shows an example of the FASTA sequence format recognized by Sequence-ID.

137 The user can copy the FASTA sequence and paste it in the "Blast" tab text form to perform a

138 BLAST search on the example query, or can download the database in FASTA format. After the

139 BLAST is performed, the web-page will provide a table including the BLAST search results for 140 the query of interest using the BLAST alignment format.

141 Genotype-ID. Genotype-ID is a web application designed to be user-friendly, and to

142 facilitate the interaction with $\mathrm{R}$ using the set of tools listed in Table 1 (Figure 1). To develop the

143 web application, we used the R package 'shiny' (Rstudio team, 2015). Shiny facilitates the

144 interactions between user, server, and R (Figure 1). The shiny web framework relies on reactive

145 programming, which allows dynamic deployment of traditional R scripts in response to data

146 input through a website console whereby results generated in R are subsequently pushed to the

147 end user. Thus, ordinary R packages, which otherwise require familiarity with the language, can

148 be deployed behind a user-friendly interface. Genotype-ID interacts principally with the R

149 packages 'poppr’ (Kamvar et al., 2014), ’adegenet` (Jombart, 2008), ’ape` (Paradis et al, 2004),

150 and 'pegas` (Paradis, 2010) (Table 1).

151 Genotype-ID has three different modules, which are shown as tabs on the website, each

152 specific to different molecular markers: SSR/microsatellite data (SSR/Microsatellite data),

153 multilocus genotype sequence types (MLST data), and AFLP, RFLP, SNP and other binary

154 datasets (Binary (AFLP) data). The user supplies a query via the web framework that is read into

$155 \mathrm{R}$ and queried against a curated dataset. Genetic distances (Table 2) are calculated between the

156 query and genotypes in the reference database, with relationship of the query to the database 
157 presented either as a UPGMA or neighbor-joining dendrogram with bootstrap support or a

158 minimum spanning network. These methods reconstruct relationships of the query relative to the

159 genotypes found in the curated database. Each module has its own customization scheme to

160 analyze each particular molecular marker type. The SSR data tool calculates Bruvo's distance

161 (Bruvo et al. 2004) to reconstruct the UPGMA or NJ dendrogram and minimum spanning

162 networks. The MLST data tool permits the comparison of user-submitted gene sequences in

163 FASTA format. The MLST data tool uses MAFFT (Katoh et al. 2002) to align each query

164 sequence to the corresponding curated gene database. It then concatenates all separate

165 alignments, calculates the genetic distance for the alignment, and reconstructs distance

166 dendrograms and minimum spanning networks. Lastly, the binary tool uses molecular markers

167 such as AFLPs or RFLPs to reconstruct relationships. This tool uses binary data (coded as 1 and

168 0) and different genetic distances (Table 2) to reconstruct the UPGMA or NJ dendrogram and the

169 minimum spanning network.

170

The Microbe-ID implementation. Microbe-ID contains implementations of each of the

172 modules for different custom databases: the SSR implementation uses nine diploid SSR loci for

173 the oomycete species P. ramorum (Supplementary Figure 2); the MLST implementation uses

174 eight multilocus sequence types for the bacterial species Clavibacter michiganensis subsp.

175 michiganensis; and a binary implementation that uses 56 loci for AFLP data for the oomycete

176 Aphanomyces euteiches. Each of the implementations of Genotype-ID contains collapsible

177 instructions on how to format the query and a link to download a tabulated file with example

178 queries formatted for use in Genotype-ID. The user can download the spreadsheet in order to

179 edit, copy, and paste their custom queries into the "Data input" form. To make analyses that use 
180 a random seed repeatable, the user has the option to specify a seed number. In MLST-ID and

181 Binary-ID, the user can select the genetic distance to be used in the analysis. After the user

182 inputs the query and runs the web application, the web page will proceed to the Analysis section,

183 where the user can choose between two different visualizations, a distance tree with bootstrap

184 support values or a minimum spanning network. If the distance tree is selected, the user can

185 change the tree algorithm (either neighbor joining or UPGMA) and number of bootstrap

186 replicates. The user can also download results as a PDF or in NEWICK format. For the minimum

187 spanning network, the user can adjust the grey scale for edge distances and download results as a

188 PDF file. Implementations for other genetic data and different visualizations can be added.

Phytophthora-ID implementation. Tools provided in Microbe-ID were implemented in

190 http://Phytophthora-ID.org, a functional website for identifying samples from the genus

191 Phytophthora, a group of economically important plant pathogens in the stramenopile branch of

192 the tree of life (Kamoun et al. 2015). Phytophthora-ID version 1.0 (Grünwald et al. 2011) had a

193 BLAST script to identify samples using the ITS barcode. Phytophthora-ID version 2.0 was

194 substantially revised and upgraded from the first iteration and now includes a faster BLAST

195 search implemented in Sequence-ID and a new genotype identification system implemented in

196 Genotype-ID.

197 The current version of Phytophthora-ID contains a Sequence-ID module customized for

198 two molecular barcodes used for Phytophthora species identification (ITS and cox spacer). This

199 particular version of the sequence identification tool was created in PERL-CGI, and permits the

200 search of a FASTA sequence query against a curated database of Phytophthora species. The

201 PERL-CGI for Phytophthora-ID version 2.0 was also redesigned to run directly on the server to

202 make the web application more stable and faster. In contrast, in Phytophthora-ID 1.0, the web 
203 application was designed as a communication wrapper to an external cluster, thus rendering 204 functionality dependent on the external server.

205 We implemented the Sequence-ID module to use sequences from two genetic regions: the 206 nuclear internal transcribed spacer (ITS) and mitochondrial cox spacer region spanning the cox 1 207 and cox2 loci. Databases were created using sequences from published Phytophthora species 208 descriptions (Blair et al., 2008; Martin and Tooley, 2003) or those that belong to classical 209 Phytophthora species described at least 25 years ago and are still recognized as valid (Erwin and 210 Ribeiro, 1996).

211 For the ITS region, we gathered a total of 211 sequences representing 108 species. For

212 the cox spacer region, we created a database of 150 sequences representing 106 species.

213 Laboratory protocols for preparing samples for sequence analysis are available on the website 214 and in a previous publication (Grünwald et al., 2011). Additionally, a file with the complete set 215 of ITS sequences, cox spacer sequences, GenBank accession numbers, and Phytophthora spp. 216 can be downloaded from the website. Documentation on updates to the databases is provided on 217 the website.

218 We implemented two modules for genotype identification of two Phytophthora species:

$219 P$. infestans that causes potato late blight, and P. ramorum that causes sudden oak death. $P$.

220 infestans has more than 18 reported clonal lineages (Hu et al., 2012), while P. ramorum has 4

221 reported clonal lineages (Grünwald et al., 2009; Van Poucke et al., 2012). To establish a database

222 with a wide representation of clonal lineages in each species, we obtained and prepared DNA

223 samples from $P$. infestans and $P$. ramorum that were collected from various regions of the world.

224 A total of 48 P. ramorum isolates representing the 4 reported clonal linages (NA1, NA2, EU1,

225 EU2) were genotyped at nine SSR loci (Prospero et al., 2007; Vercauteren et al., 2010; Grünwald 
226 et al., 2009; Ivors et al., 2006). Similarly, 11 P. infestans clonal lineages that are dominant in the

227 US (including US11, US12, US8, US20, US21, US23, US24, EU4, EU5, EU8, EU13)

228 represented by a total population of 96 isolates were genotyped at 11 SSR loci, using protocols

229 from Lees et al. (2006) and Li et al. (2013). We constructed SSR reference databases compatible

230 with the poppr/adegenet $\mathrm{R}$ packages that provide dendrograms and minimum spanning networks

231 for queries. Bootstrap support can be calculated for dendrograms. To test the datasets, we used

232 two queries per dataset, one sample of NA1 and NA2 clonal lineages each for P. ramorum and

233 one sample of clonal lineages US-23 and US-8 clonal lineages for P. infestans. All samples

234 grouped with the corresponding reference lineages with high support values (Figures 2 and 3),

235 demonstrating functionality of the Microbe-ID tool to identify samples correctly.

237 Conclusions

238 We constructed a web framework that can use a wide array of molecular markers to

239 rapidly determine identities of species and genotypes. This web framework is provided as open

240 source code on github (https://github.com/grunwaldlab/Microbe-ID). We implemented a

241 demonstration available at http://Microbe-ID.org. New implementations for any organisms

242 require reference databases, scripts, and web pages including choices from the set of

243 computational tools shown in Table 1 . The Microbe-ID framework is currently implemented for

244 two fully functional sites, Phytophthora-ID (http://Phytophthora-ID.org/) and Gall-ID

245 (http://Gall-ID.cgrb.oregonstate.edu/; Davis et al. 2016).

246 Modularity of Microbe-ID permits implementation of a range of markers using curated

247 databases of known species or genotypes to determine identity of specimens. Use of a custom

248 BLAST database permits identification of any sample of a species of interest by using any 
249 sequence-based molecular marker. Note that the amount of time required for BLAST execution

250 is dependent on size of the databases. For the Sequence-ID ITS region implementation for

251 Phytophthora, each run was completed and loaded to the web-app in less than one second, with

252 results shown as a BLAST output table.

253 Most innovative in this web framework is the Genotype-ID module, which permits the

254 researcher to use, in a simple, web-based interface, any type of molecular marker with a

255 corresponding and curated reference dataset. Use of a shiny server for strain identification is

256 completely novel and modularity permits use of virtually any molecular marker suitable for

257 existing R packages. For genotyping, population genetic markers demonstrated include SSR,

258 MLST, and AFLP/RFLP data, but can also be expanded to presence/absence of genes or alleles

259 or any other genetic features of interest. Custom implementations must include a reference

260 dataset and custom R scripts. Implementations can be customized for virtually any organism and

261 any molecular marker that can be analyzed in R.

262 Development of tools for species identification has increased in recent years; but lack of

263 modularity and complicated methodological procedures make the process of species

264 identification tedious and challenging for researchers without sufficient skills in computational

265 biology. We developed an easy-to-set-up web framework, which permits quick and flexible

266 deployment of species identification and genotyping tools using sequence, microsatellite/SSR,

267 AFLP/RFLP, or MLST data for any organism. Given use of the bootstrap html framework,

268 webpages are light in terms of computer resources required and are thus usable on any device

269 including smartphones and tablets, and function with any browser. These tools have been

270 deployed and used successfully for the genus Phytophthora and gall-forming bacteria, providing

271 examples of two curated websites (Grünwald et al. 2011; Davis et al. 2016). 
273

274

275

276

277

278

279

280

281

282

283

284

285

286

287

288

289

290

291

292

293

\section{References}

Aurrecoechea, C., Barreto, A., Brestelli, J., Brunk, B.P., Cade, S., Doherty, R., Fischer, S., Gajria, B., Gao, X., Gingle, A. \& Grant, G. (2012). EuPathDB: The eukaryotic pathogen database. Nucleic Acids Research 41, D684-D691.

Altschul, S.F., Gish, W., Miller, W., Myers, E.W. \& Lipman, D.J. (1990). "Basic local alignment search tool." Journal of Molecular Biology 215,403-410.

Blair, J.E., Coffey, M.D., Park, S.-Y., Geiser, D.M., \& Kang, S. (2008). A multi-locus phylogeny for Phytophthora utilizing markers derived from complete genome sequences. Fungal Genetics and Biology 45, 266-277.

Bruvo, R., Michiels, N.K., D’Souza, T.G., \& Schulenburg, H. (2004). A simple method for the calculation of microsatellite genotype distances irrespective of ploidy level. Molecular Ecology, 13, 2101-2106.

Byrnes III, E.J., Li, W., Lewit, Y., Ma, H., Voelz, K., Ren, P., Carter, D.A., Chaturvedi, V., Bildfell, R.J., May, R.C. and Heitman, J. (2010). Emergence and pathogenicity of highly virulent Cryptococcus gattii genotypes in the northwest United States. PLoS Pathogens 6(4), p.e1000850.

Coleman, A.W. (2003). ITS2 is a double-edged tool for eukaryote evolutionary comparisons. Trends in Genetics 19, 370-355.

Coleman, A.W. (2007). Pan-eukaryote ITS2 homologies revealed by RNA secondary structure. Nucleic Acids Research 35, 3322-3329. 
294 Coleman, A.W. (2009). Is there a molecular key to the level of "biological species" in 295 eukaryotes? A DNA guide. Molecular Phylogenetics and Evolution 50, 197-203.

296 Cooke, D.E., Cano, L.M., Raffaele, S., Bain, R.A., Cooke, L.R., Etherington, G.J., Deahl, K.L., 297 Farrer, R.A., Gilroy, E.M., Goss, E.M. \& Grünwald, N.J. (2012). Genome analyses of an 298 299

Davis, E.W., Weisberg, A.J., Tabima, J.F., Grünwald, N.J., \& Chang, J.H. (2016). Gall-ID:

301

302

303 aggressive and invasive lineage of the Irish potato famine pathogen. PLoS Pathogens 8, e1002940. Tools for genotyping gall-causing phytopathogenic bacteria. PeerJ Preprints 4, e1998v1. DOI: 10.7287/peerj.preprints.1998v1.

Edwards, A.W.F. (1971). Distances between populations on the basis of gene frequencies. Biometrics 27, 873-881.

Elshire, R.J., Glaubitz, J.C., Sun, Q., Poland, J.A., Kawamoto, K., Buckler, E.S., \& Mitchell, S.E. (2011). A robust, simple genotyping-by-sequencing (GBS) approach for high diversity species. PLoS ONE 6, e19379.

Erwin, D.C. and Ribeiro, O.K., (1996). Phytophthora diseases worldwide. American Phytopathological Society, St. Paul, MN (USA).

Etter, P.D., Bassham, S., Hohenlohe, P.A., Johnson, E.A. and Cresko, W.A. (2010). SNP discovery and genotyping for evolutionary genetics using RAD sequencing. In Methods in Molecular Biology (Clifton, NJ), 772, 157-178.

Felsenstein, J. (1981). Evolutionary trees from DNA sequences: A maximum likelihood approach. Journal of Molecular Evolution 17, 368-376.

Felsenstein, J. (1989). PHYLIP - Phylogeny Inference Package (Version 3.2). Cladistics 5, 164166. 
317 Grajales, A., Aguilar, C., \& Sánchez, J.A. (2007). Phylogenetic reconstruction using secondary

318 structures of Internal Transcribed Spacer 2 (ITS2, rDNA): Finding the molecular and

319 morphological gap in Caribbean gorgonian corals. BMC Evolutionary Biology 7, 90.

320 Grigoriev, I.V., Nikitin, R., Haridas, S., Kuo, A., Ohm, R., Otillar, R., Riley, R., Salamov, A.,

321 Zhao, X., Korzeniewski, F. \& Smirnova, T. (2013). MycoCosm portal: Gearing up for

3221000 fungal genomes. Nucleic Acids Research 42, D699-D704.

323 Gross A, Holdenrieder O, Pautasso M, Queloz V, \& Sieber TN. (2014). Hymenoscyphus

324 pseudoalbidus, the causal agent of European ash dieback. Molecular Plant Pathology 1,

$325 \quad 5-21$.

326 Grünwald, N.J., Goss, E.M., Ivors, K., Garbelotto, M., Martin, F.N., Prospero, S., Hansen, E.,

327 Bonants, P.J., Hamelin, R.C., Chastagner, G. \& Werres, S. (2009). Standardizing the

328 nomenclature for clonal lineages of the sudden oak death pathogen, Phytophthora

329 ramorum. Phytopathology 99, 792-795.

330 Grünwald, N.J., Martin, F.N., Larsen, M.M., Sullivan, C.M., Press, C.M., Coffey, M.D., Hansen,

331 E.M., \& Parke, J.L. (2011). Phytophthora-ID.org: A sequence based Phytophthora

332 identification tool. Plant Disease 95, 337-342.

333 Grünwald, N. J., McDonald, B. M., \& Milgroom, M. G. (2016). Population genomics of fungal

334 and oomycete pathogens. Annual Review of Phytopathology, in press.

335 Grünwald, N.J., Goss, E.M. \& Press, C.M. (2008). Phytophthora ramorum: A pathogen with a

336 remarkably wide host-range causing sudden oak death on oaks and ramorum blight on

337 woody ornamentals. Molecular Plant Pathology 9, 729-740. 
338 Grünwald, N.J., \& Hoheisel, G.-A. (2006). Hierarchical analysis of diversity, selfing and genetic 339 differentiation in populations of the oomycete Aphanomyces euteiches. Phytopathology $96,1134-1141$.

341 Holdich, D.M., Reynolds, J.D., Souty-Grosset, C. \& Sibley, P.J. (2009). A review of the ever increasing threat to European crayfish from non-indigenous crayfish species. Knowledge and Management of Aquatic Ecosystems 11, 394-395.

Hu, C.H., Perez, F.G., Donahoo, R., McLeod, A., Myers, K., Ivors, K., Secor, G., Roberts, P., Deahl, K.L., Fry, W.E. \& Ristaino, J.B. (2012). Recent genotypes of Phytophthora infestans in the eastern United States reveal clonal populations and reappearance of mefenoxam sensitivity. Plant Disease 96, 1323-1330.

Huang, X., Feng, Q., Qian, Q., Zhao, Q., Wang, L., Wang, A., Guan, J., Fan, D., Weng, Q., Huang, T. and Dong, G. (2009). High-throughput genotyping by whole-genome resequencing. Genome Research 19, 1068-1076.

Ivors, K., Garbelotto, M., Vries, I.D.E., Ruyter-Spira, C., Te Hekkert, B., Rosenzweig, N., \& Bonants, P. (2006). Microsatellite markers identify three lineages of Phytophthora ramorum in US nurseries, yet single lineages in US forest and European nursery Bioinformatics 24, 1403-1405. 
360 Jukes, T.H. and Cantor, C.R. (1969). Evolution of protein molecules: Mammalian Protein $361 \quad$ Metabolism 3, 132.

362 Kamoun, S., Furzer, O., Jones, J.D.G., Judelson, H.S., Shad Ali, G., Dalio, R.J.D., Guha Roy, S., 363 Schena, L., Zampounis, A., Panabières, F., Cahill, D., Ruocco, M., Figueiredo, A., Chen,

Katoh, K., Misawa, K., Kuma, K. I., \& Miyata, T. (2002). MAFFT: A novel method for rapid multiple sequence alignment based on fast Fourier transform. Nucleic Acids Research 30, 3059-3066.

Kimura, M. (1980). A simple method for estimating evolutionary rates of base substitutions through comparative studies of nucleotide sequences. Journal of Molecular Evolution. 16, $111-120$.

Klevens RM, Morrison MA, Nadle J, Petit S, Gershman K, Ray S, Harrison LH, Lynfield R, Dumyati G, Townes JM, \& Craig AS. (2007). Invasive methicillin-resistant Staphylococcus aureus infections in the United States. Jama 298, 1763-71. 
382 Kroon, L.P., Brouwer, H., de Cock, A.W. \& Govers, F. (2012). The genus Phytophthora anno 383 2012. Phytopathology 102, 348-364.

384 Lees, A.K., Wattier, R., Shaw, D.S., Sullivan, L., Williams, N.A., \& Cooke, D.E.L. (2006).

385

386

387

388

389

390

391

392

393

394

395

396

397

398

399

400

401

402

403

404 Novel microsatellite markers for the analysis of Phytophthora infestans populations. Plant Pathology 55, 311-319.

Li, Y., Cooke, D.E.L., Jacobsen, E., \& van der Lee, T. (2013). Efficient multiplex simple sequence repeat genotyping of the oomycete plant pathogen Phytophthora infestans. Journal of Microbiological Methods 92, 316-322.

Maiden, M.C.J., van Rensburg, M.J.J., Bray, J.E., Earle, S.G., Ford, S.A., Jolley, K.A., \& McCarthy, N.D. (2013). MLST revisited: the gene-by-gene approach to bacterial genomics. Nature Reviews Microbiology 11, 728-736.

Martin, F.N., \& Tooley, P.W. (2003). Phylogenetic relationships among Phytophthora species inferred from sequence analysis of mitochondrially encoded cytochrome oxidase I and II genes. Mycologia 95, 269-284.

Nei, M. (1972). Genetic distance between populations. The American Naturalist 106, 283-292.

Paradis, E., Claude, J., \& Strimmer, K. (2004). APE: Analyses of phylogenetics and evolution in R language. Bioinformatics 20, 289-290.

Paradis E. (2010). Pegas: An R package for population genetics with an integrated-modular approach. Bioinformatics 26, 419-420

Park, J., Park, B., Veeraraghavan, N., Jung, K., Lee, Y.-H., Blair, J. E., Geiser, D. M., Isard, S., Mansfield, M. A., Nikolaeva, E., Park, S.-Y., Russo, J., Kim, S. H., Greene, M., Ivors, K. L., Balci, Y., Peiman, M., Erwin, D. C., Coffey, M. D., Rossman, A., Farr, D., Cline, E., Grünwald, N. J., Luster, D. G., Schrandt, J., Martin, F., Ribeiro, O. K., Makalowska, I., 
405

406

407

408

409

410

411

412

413

414

415

416

417

418

419

420

421

422

423

424

425

426

and Kang, S. (2008). Phytophthora Database: A forensic database supporting the identification and monitoring of Phytophthora. Plant Disease 92, 966-972.

Prevosti, A., Ocaña, J., \& Alonso, G. (1975). Distances between populations of Drosophila subobscura, based on chromosome arrangement frequencies. Theoretical and Applied Genetics 45, 231-241.

Prospero, S., Hansen, E.M., Grünwald, N.J., \& Winton, L.M. (2007). Population dynamics of the sudden oak death pathogen Phytophthora ramorum in Oregon from 2001 to 2004. Molecular Ecology 16, 2958-2973.

Reynolds, J., Weir, B.S., \& Cockerham, C.C. (1983). Estimation of the coancestry coefficient: basis for a short-term genetic distance. Genetics 105, 767-779.

Robideau, G.P., de Cock, A.W., Coffey, M.D., Voglmayr, H., Brouwer, H., Bala, K., Chitty, D.W., Désaulniers, N., Eggertson, Q.A. \& Gachon, C.M.M. (2011). DNA barcoding of oomycetes with cytochrome c oxidase subunit I and internal transcribed spacer. Molecular Ecology Resources 11, 1002-1011.

Rogers, J.S. (1972). Measures of genetic similarity and genetic distance. Studies in Genetics 7 , 145-153.

RStudio Team (2015). RStudio: Integrated Development for R. RStudio, Inc., Boston, MA URL http://www.rstudio.com/.

Schoch, C.L., Seifert, K.A., Huhndorf, S., Robert, V., Spouge, J.L., Levesque, C.A., \& Chen, W. (2012). Nuclear ribosomal internal transcribed spacer (ITS) region as a universal DNA barcode marker for Fungi. Proceedings of the National Academy of Sciences of the United States of America 109, 6241-6246. 
427 Stajich, J.E., Harris, T., Brunk, B.P., Brestelli, J., Fischer, S., Harb, O.S., Kissinger, J.C., Li, W., 428 Nayak, V., Pinney, D.F. \& Stoeckert, C.J., (2011). FungiDB: An integrated functional 429 genomics database for fungi. Nucleic Acids Research 40, D675-81.

430 Tamura, K., \& Nei, M. (1993). Estimation of the number of nucleotide substitutions in the 431 control region of mitochondrial DNA in humans and chimpanzees. Molecular Biology and Evolution 10, 512-526.

433 Tancos, M.A., Lange, H.W., \& Smart, C.D. (2015). Characterizing the genetic diversity of the $434 \quad$ Clavibacter michiganensis subsp. michiganensis population in New York.

435 Phytopathology 105, 169-179.

436 Van Poucke, K., Franceschini, S., Webber, J.F., Vercauteren, A., Turner, J.A., McCracken, A.R., 437 Heungens, K., \& Brasier, C.M. (2012). Discovery of a fourth evolutionary lineage of 438 Phytophthora ramorum: EU2. Fungal Biology 116, 1178-1191.

439 Vercauteren, A., De Dobbelaere, I., Grünwald, N.J., Bonants, P., Van Bockstaele, E., Maes, M., 440 \& Heungens, K. (2010). Clonal expansion of the Belgian Phytophthora ramorum populations based on new microsatellite markers. Molecular Ecology 19, 92-107. 


\section{Figure Legends}

443 Figure 1. Diagram representing implementation of Genotype-ID, which is comprised of a user

444 interface file (index.html) and a server file (server.R). Each file communicates with the R

445 framework (via shiny) and user (via HTML5). On the user side (left side), user input is provided

446 by copy/paste of a query and selects/specifies the desired application modifiers (seed number,

447 genetic distance calculation). This information is subsequently received and processed by the

448 server file, prompting the application to run in R. On the server side (right side) a database file

449 (Marker DB), R packages, and functions are retrieved and executed. When the run is complete,

450 the server file provides output to the user interface file and displayed on the app output.

451

452 Figure 2. Results of SSR-ID for NA1 and NA2 queries of $P$. ramorum provided in the example 453 data file. Each color represents a clonal lineage pre-assigned to each reference sample (NA1, 454 NA2, EU1, EU2) with queries colored in red. A. UPGMA tree with 1,000 bootstrap replicates 455 and support values above branches. Queries are represented in red and all are correctly placed 456 with reference samples of the presumptive clonal lineage while also representing the relationship

457 between clonal lineages in the reference dataset. B. Minimum spanning network reconstruction. 458 Edge shade and width are inversely proportional to Bruvo's distance as shown in the horizontal 459 scale bar. Queries are represented in red and placed in nodes with the most similar reference 460 sample in the dataset, indicating the NA1 query is most similar to the PR-12-044 reference 461 sample and the NA2 query is more closely related to the PR-05-156 and PR-12-103 samples, 462 which also belong to the NA2 clonal lineage. 463 
464 Figure 3. Results of SSR-ID queries for strains placed into the US8 and US23 clonal lineages of 465 the potato late blight pathogen, $P$. infestans. Colors correspond to clonal lineages assigned to 466 each reference sample (B, C, EU-13, EU-14, etc.) except for the queries which are colored in red. 467 A. UPGMA tree with 1,000 bootstrap replicates with support values above branches. Queries are 468 represented in red and all are correctly placed with samples of the presumptive clonal lineage 469 while also representing relationships between clonal lineages in the reference dataset. B.

470 Minimum spanning network reconstruction. Edge shade and width are proportional to Bruvo's 471 distance shown in the horizontal scale bar. Queries are represented in red nodes and appear in 472 legend as '???'. Queries placed in nodes with the most similar reference sample, indicating that 473 the US8 query is most similar to the PI-12-016 reference sample (US-8 clonal lineage) and the 474 US23 query is most closely related to the PI-12-023 sample, part of the US-23 lineage. 


\section{Supplementary Figures}

476

477 Supplementary Figure 1. Screen capture of the Sequence-ID user interface of Microbe-ID. The 478 interface includes tabs for input of barcodes and a dropdown help link that contains laboratory 479 protocols, examples data, and logs of each query dataset. The input frame has a control scheme 480 that only permits data in FASTA format.

481

482 Supplementary Figure 2. Screen capture of the Genotype-ID user interface of Microbe-ID.

483 Shown is the SSR-ID module for P. ramorum. Genotype-ID includes an Instructions link with an 484 example Excel file that the user can modify, copy, and paste into the data input form. There is no 485 limit to the number of queries submitted. In the Analysis section, tabs are provided to select 486 either between a distance tree or a minimum spanning network rendering.

Supplementary Figure 3. UPGMA dendrogram of sub-module MLST-ID of Genotype-ID. The tree was constructed using 100 bootstrap replicates and MLST analysis of 8 genes ( 5 housekeeping genes and 3 virulence genes) of Clavibacter michiganensis. The query used is a concatenation of a WASH sample using all 8 genes. Queries are represented in blue. Note that all queries are correctly placed amongst samples of its presumptive clonal lineage while also representing relationships between lineages in the reference dataset.

Supplementary Figure 4. UPGMA dendrogram of sub-module Binary-ID of Genotype-ID using 100 bootstrap replicates for Aphanomyces euteiches. The queries used for this iteration of Binary-ID are two samples from a presumptive "Athena" origin. Queries are represented in red. 
498 Note that all queries were correctly placed amongst samples of its presumptive clonal lineage

499 while also representing relationships between lineages in the reference dataset, indicating both 500 queries are more closely related to the "Athena" population than the "Mt. Vernon" population. 


\section{Figure 1 (on next page)}

\section{Figure 1}

Figure 1. Diagram representing implementation of Genotype-ID, which is comprised of a user interface file (index.html) and a server file (server.R). Each file communicates with the $\mathrm{R}$ framework (via shiny) and user (via HTML5). On the user side (left side), user input is provided by copy/paste of a query and selects/specifies the desired application modifiers (seed number, genetic distance calculation). This information is subsequently received and processed by the server file, prompting the application to run in R. On the server side (right side) a database file (Marker DB), R packages, and functions are retrieved and executed. When the run is complete, the server file provides output to the user interface file and displayed on the app output. 


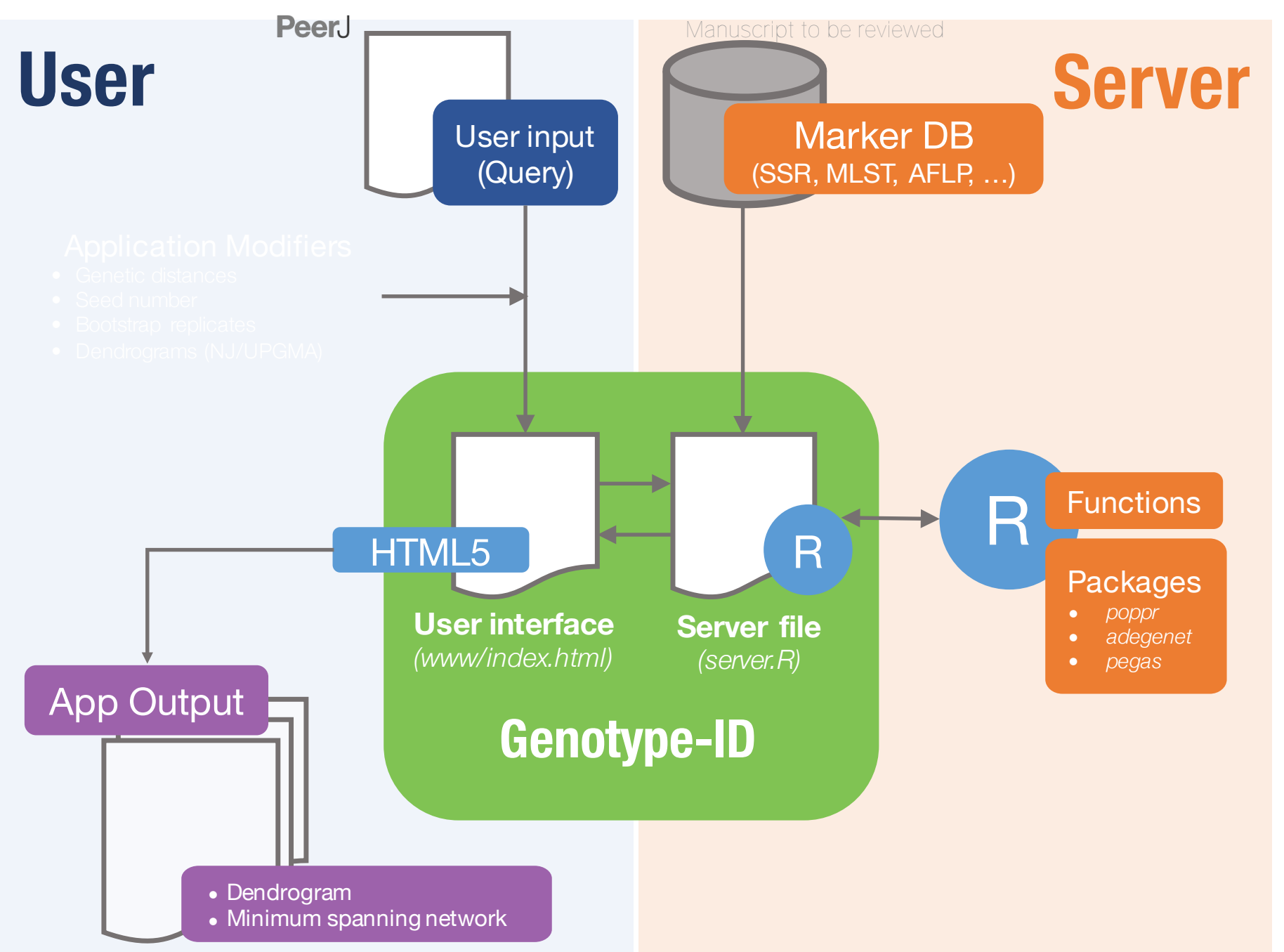




\section{Figure 2 (on next page)}

Figure 2

Figure 2. Results of SSR-ID for NA1 and NA2 queries of $P$. ramorum provided in the example data file. Each color represents a clonal lineage pre-assigned to each reference sample (NA1, NA2, EU1, EU2) with queries colored in red. A. UPGMA tree with 1,000 bootstrap replicates and support values above branches. Queries are represented in red and all are correctly placed with reference samples of the presumptive clonal lineage while also representing the relationship between clonal lineages in the reference dataset. B. Minimum spanning network reconstruction. Edge shade and width are inversely proportional to Bruvo's distance as shown in the horizontal scale bar. Queries are represented in red and placed in nodes with the most similar reference sample in the dataset, indicating the NA1 query is most similar to the PR-12-044 reference sample and the NA2 query is more closely related to the PR-05-156 and PR-12-103 samples, which also belong to the NA2 clonal lineage. 
A.
0.1

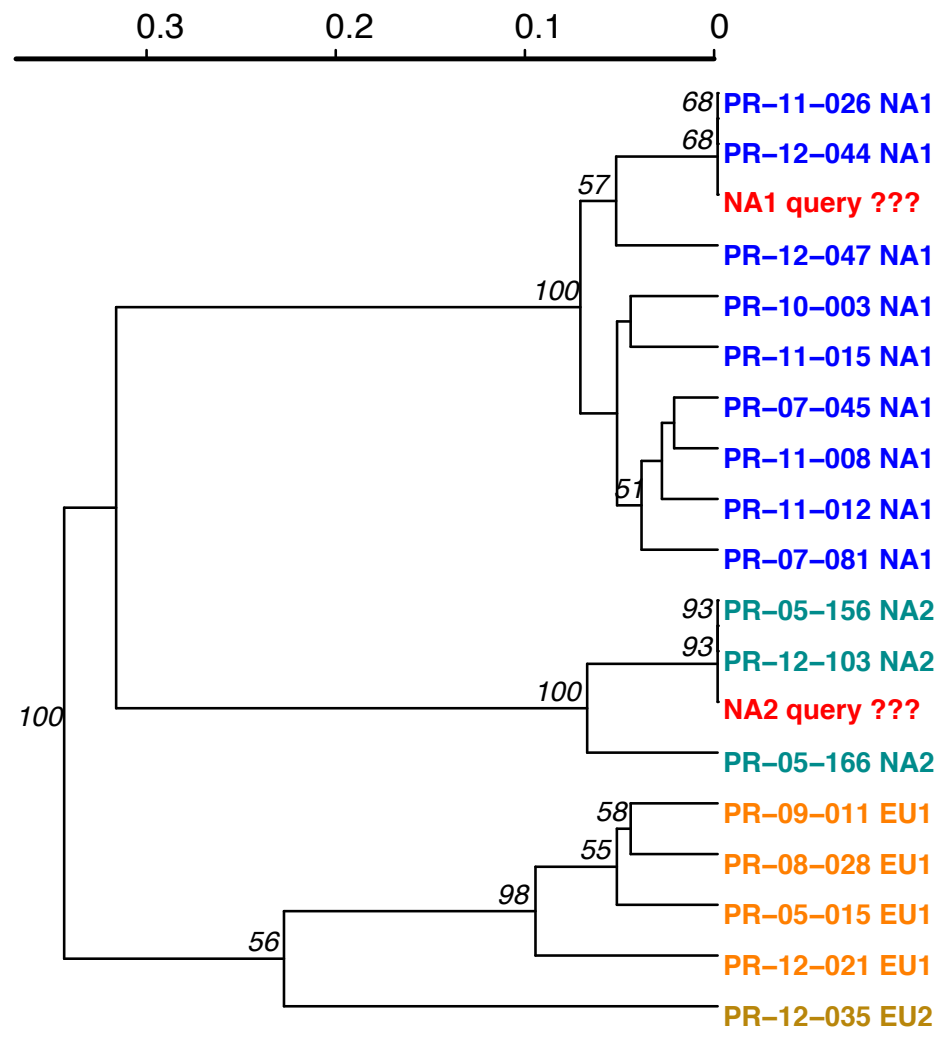

B.

Manuscript to be reviewed

\section{POPULATION}

$\square$ NA1

$\square$ NA2

$\square$ EU1

$\square$ EU2

$\square$ Query
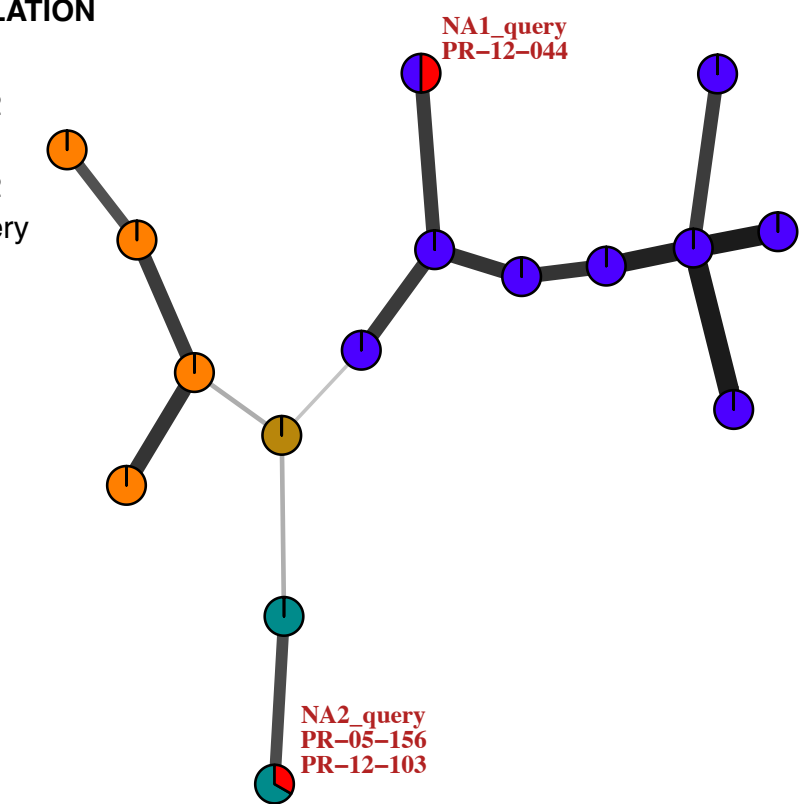

0.047

0.197

0.346

0.496

0.646 


\section{Figure 3 (on next page)}

Figure 3

Figure 3. Results of SSR-ID queries for strains placed into the US8 and US23 clonal lineages of the potato late blight pathogen, $P$. infestans. Colors correspond to clonal lineages assigned to each reference sample (B, C, EU-13, EU-14, etc.) except for the queries which are colored in red. A. UPGMA tree with 1,000 bootstrap replicates with support values above branches. Queries are represented in red and all are correctly placed with samples of the presumptive clonal lineage while also representing relationships between clonal lineages in the reference dataset. B. Minimum spanning network reconstruction. Edge shade and width are proportional to Bruvo's distance shown in the horizontal scale bar. Queries are represented in red nodes and appear in legend as '???'. Queries placed in nodes with the most similar reference sample, indicating that the US8 query is most similar to the PI-12-016 reference sample (US-8 clonal lineage) and the US23 query is most closely related to the PI12-023 sample, part of the US-23 lineage. 


\section{A. \\ $\mathbf{B}^{\text {Manuscript to be reviewed }}$}

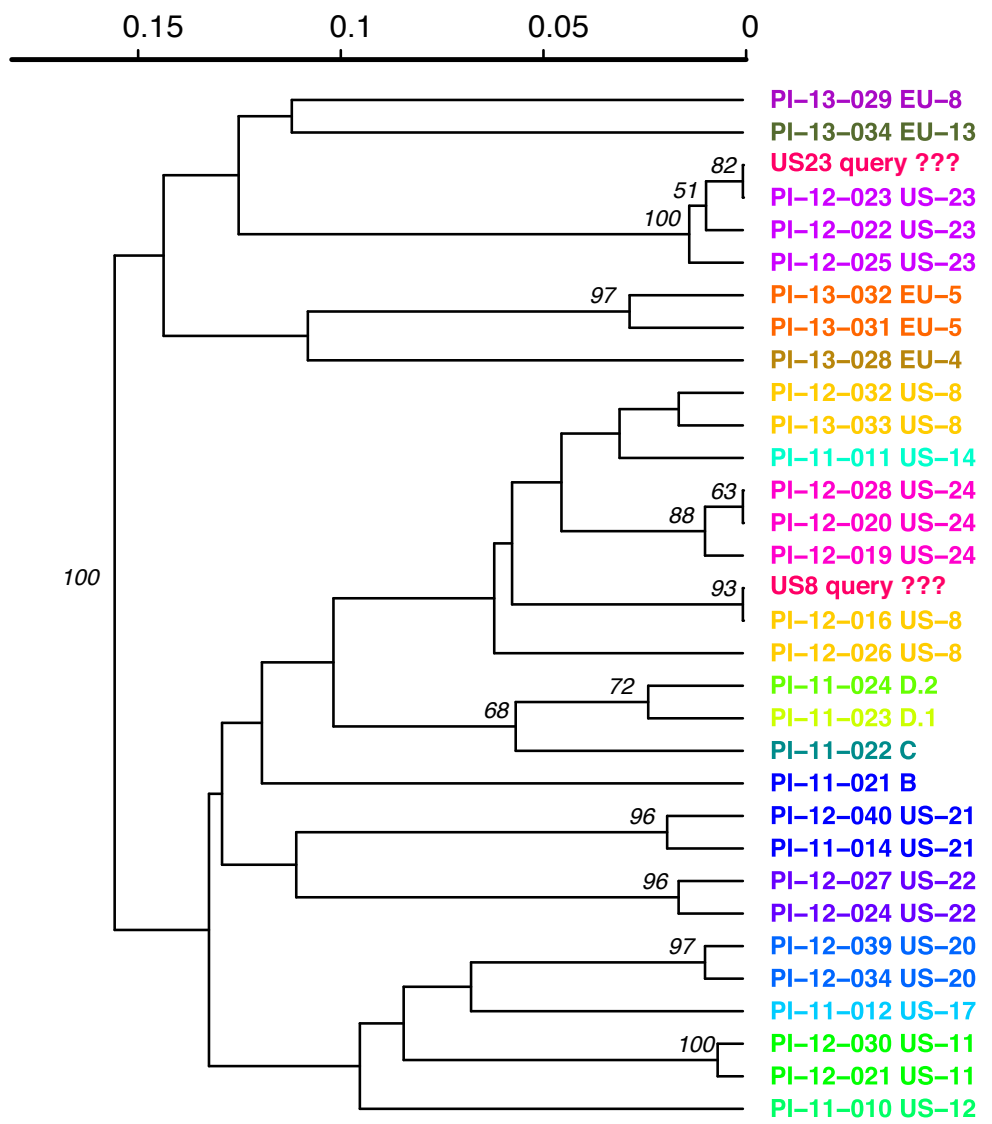

\section{POPULATION}

$\square$ B

$\square \quad C$

- EU-13

$\square$ EU-4

EU-8

$\square$ EU-5

$\square$ US-8

$\square$ D.1

$\square$ D.2

$\square$ US-11

$\square$ US-12

$\square$ US-14

$\square$ US-17

ㄴ US-20

- US-21

$\square$ US-22

- US-23

$\square$ US-24

???

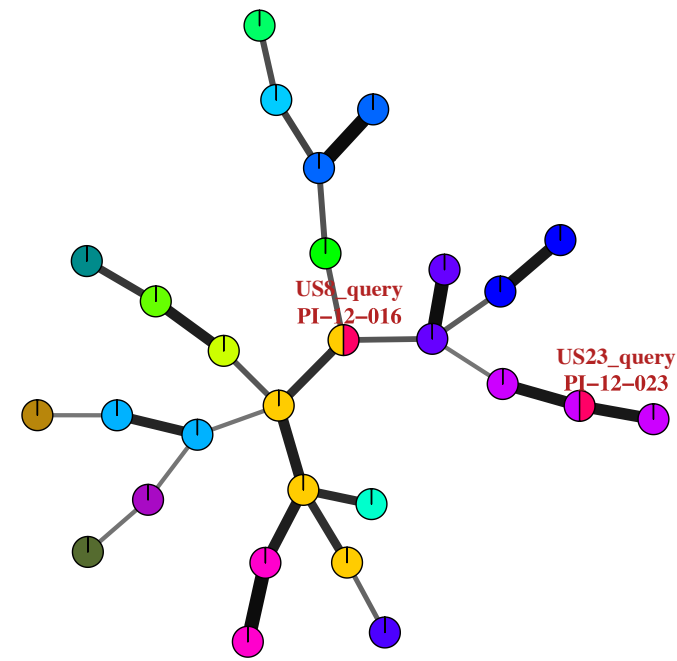

0.014

0.073

0.132

0.191

0.251

DISTANCE 


\section{Table $\mathbf{1}$ (on next page)}

Table 1 
1 Table 1. Open source computational tools required to install and deploy Microbe-ID on a server.

2 - = reference not available; see website provided for information.

\begin{tabular}{|c|c|c|c|}
\hline Tool & Description & Source & Reference \\
\hline Ape & $\begin{array}{l}\text { R package for phylogenetic and } \\
\text { evolutionary analysis }\end{array}$ & $\underline{\text { http://ape-package.ird.fr/ }}$ & $\begin{array}{l}\text { Paradis et al., } \\
2004\end{array}$ \\
\hline$B L A S T$ & $\begin{array}{l}\text { Basic Local Alignment and } \\
\text { Search Tool implemented as an } \\
\text { algorithm for comparing DNA, } \\
\text { RNA or protein query sequences } \\
\text { against a reference database }\end{array}$ & $\begin{array}{l}\frac{\mathrm{ftp}: / / \mathrm{ftp} . n c b i . n l m . n i h . g o v / \mathrm{bl}}{\text { ast/executables/blast+/LAT }} \\
\underline{\text { EST/ }}\end{array}$ & $\begin{array}{l}\text { Altschul et } \\
\text { al., } 1990\end{array}$ \\
\hline Adegenet & $\begin{array}{l}\mathrm{R} \text { package for multivariate } \\
\text { analysis of genetic data }\end{array}$ & $\begin{array}{l}\text { https://github.com/thibautjo } \\
\text { mbart/adegenet/ }\end{array}$ & $\begin{array}{l}\text { Jombart, } \\
2008\end{array}$ \\
\hline Bootstrap & $\begin{array}{l}\text { A framework for developing } \\
\text { responsive, mobile-first projects } \\
\text { on the web }\end{array}$ & $\underline{\underline{\mathrm{http}}: / / \text { www.getbootstrap.co }}$ & - \\
\hline Microbe-ID & $\begin{array}{l}\text { Set of web-apps for } \\
\text { identification of species, } \\
\text { genotypes, and strains of any } \\
\text { organism }\end{array}$ & $\begin{array}{l}\underline{\text { https://github.com/grunwald }} \\
\underline{\text { lab/Microbe-ID }}\end{array}$ & This paper. \\
\hline$M A F F T$ & $\begin{array}{l}\text { Multiple sequence alignment } \\
\text { algorithm to find homology } \\
\text { between sequences using Fourier } \\
\text { algorithms }\end{array}$ & $\begin{array}{l}\underline{\text { http://mafft.cbrc.jp/alignme }} \\
\underline{\text { nt/software/ }}\end{array}$ & $\begin{array}{l}\text { Katoh et al., } \\
2002\end{array}$ \\
\hline Pegas & $\begin{array}{l}\text { R package for analysis of } \\
\text { population genetic data }\end{array}$ & $\begin{array}{l}\text { https://github.com/grunwald } \\
\underline{\text { lab/poppr }}\end{array}$ & $\begin{array}{l}\text { Kamvar et } \\
\text { al., 2014; } \\
\text { Kamvar et } \\
\text { al., } 2015\end{array}$ \\
\hline Poppr & $\begin{array}{l}\mathrm{R} \text { package for genetic analysis of } \\
\text { populations with mixed } \\
\text { reproduction }\end{array}$ & $\underline{\underline{\text { https://github.com/grunwald }}}$ & $\begin{array}{l}\text { Kamvar et } \\
\text { al., } 2014\end{array}$ \\
\hline Shiny & $\begin{array}{l}\text { Interactive web application } \\
\text { framework for } \mathrm{R}\end{array}$ & $\underline{\text { http://shiny.rstudio.com/ }}$ & - \\
\hline
\end{tabular}




\section{Table 2 (on next page)}

Table 2 
1 Table 2. Genetic distances implemented in the Genotype-ID module of Microbe-ID. Each of the 2 distances included in Microbe-ID are specific to a given molecular marker used in the web 3 application (MLST-ID: Multilocus sequence typing; SSR-ID: SSR/microsatellite loci; Binary4 ID: AFLP/SNP loci).

\begin{tabular}{|c|c|c|c|}
\hline Distance model & Module & R package & References \\
\hline Felsenstein 81 (F81) & MLST-ID & ape & Felsenstein (1981) \\
\hline Felsenstein 84 (F84) & MLST-ID & ape & Felsenstein (1989) \\
\hline Indel & MLST-ID & ape & Paradis et al. (2004) \\
\hline Jukes-Cantor (JC69) & MLST-ID & ape & Jukes and Cantor (1969) \\
\hline Kimura 80 (K80) & MLST-ID & ape & Kimura (1980) \\
\hline Kimura 81 (K81) & MLST-ID & ape & Kimura (1980) \\
\hline Raw & MLST-ID & ape & Paradis et al. (2004) \\
\hline Tamura and Nei 93 (TN93) & MLST-ID & ape & Tamura and Nei (1993) \\
\hline Transitions (TS) & MLST-ID & ape & Paradis et al. (2004) \\
\hline Transversions (TV) & MLST-ID & ape & Paradis et al. (2004) \\
\hline Bruvo & SSR-ID & poppr & Bruvo et al. (2004) \\
\hline Edwards & Binary-ID & poppr/adegenet & Edwards (1971) \\
\hline Nei & Binary-ID & poppr/adegenet & Nei (1972) \\
\hline Prevosti & Binary-ID & poppr/adegenet & Prevosti et al. (1974) \\
\hline Reynolds & Binary-ID & poppr/adegenet & Reynolds et al. (1983) \\
\hline Rogers & Binary-ID & poppr/adegenet & Rogers (1972) \\
\hline
\end{tabular}

